Pacific Journal of Mathematics

WHICH LINEAR MAPS OF THE DISK ALGEBRA ARE 


\title{
WHICH LINEAR MAPS OF THE DISK ALGEBRA ARE MULTIPLICATIVE?
}

\section{RICHARD ROCHBERG}

\begin{abstract}
Let $T$ be a linear map of the disk algebra into itself which is of norm one and fixes the constants. This paper considers the question of what additional restrictions suffice to insure that $T$ is multiplicative. It is shown that if $T$ is an isometry and the range of $T$ is a ring then $T$ is multiplicative and that if the image under $T$ of the coordinate function of the disk is an extreme point of the unit ball of the disk algebra then $T$ is multiplicative.
\end{abstract}

Let $D$ be the closed unit disk of the complex plane and $A$ the disk algebra, the supremum normed Banach algebra of functions continuous on $D$ and analytic in the interior of $D$. Let $Z$ be the identity function on $D, Z(x)=x$ for all $x$ in $D$. Let $L$ be the set of extreme points of the closed unit ball of $A$. Let $K$ be the set of linear maps of $A$ into itself which are of norm less than or equal one and which fix the constants. Let $K^{\prime}$ be the set of elements of $K$ which are multiplicative; $K^{\prime}=\{T ; T$ in $K, T(f g)=T(f) T(g)$ for all $f$ and $g$ in $A\}$. We note in passing that any continuous non-zero multiplicative linear map of $A$ into itself is of norm one and hence in $K^{\prime}$ and that any such map is given by composition with an element of $A$; that is if $T$ is $K^{\prime}$ then $T f=f \circ T(Z)$ for all $f$ in $A$. The first of these facts is a general function algebra result and the second follows from the fact that the polynomials are dense in $A$.

We will investigate the question of what additional restrictions are needed to insure that $T$, an element of $K$, is actually an element of $K^{\prime}$. We will prove

THEOREM A. If $T$ in $K$ is an isometry (i.e., $\|T f\|=\|f\|$ for all $A$ in $f$ ) and $T(A)$ is a ring, then $T$ is in $K^{\prime}$. and

Theorem B. If $T$ is in $K$ and $T(Z)$ is in $L$, then $T$ is in $K^{\prime}$.

Theorem A extends the known result that any surjective isometry in $K$ is multiplicative and provides a partial answer to the question raised by Phelps ([1], pg. 354) of describing the non-surjective isometries of the disk algebra.

Phelps ([3]) has shown that if $T$ is in $K^{\prime}$ and $T(Z)$ is in $L$, then $T$ is an extreme point of $K$. This result combined with Theorem $B$ gives. 
CoROLlary. If $T$ is in $K$ and $T(Z)$ is in $L$, then $T$ is an extreme point of $K$.

Examples will be given to show that the hypotheses of these two theorems cannot be eliminated and that conjectures with similar hypotheses are not true.

The reader is referred to ([2]) as a reference for the properties of the algebra $A$.

2. Isometries. If $T$ in $K$ is a surjective automorphism of $A$ then $T$ is given by composition with a conformal automorphism of $D$. A proof of this fact can be found in ([2]). The following is essentially what remains of that proof when the hypothesis of surjectivity is dropped, some of the details which are identical with that proof are omitted.

Let $C$ be the unit circle of the complex plane.

THEOREM. If $T$ in $K$ is an isometry then there is a closed set $S$ in $C$ and a function $H$ defined on $S$ to that

a. $H$ is a continuous map of $S$ onto $C$,

b. for all $z$ in $S$ and all $f$ in $A,(T f)(z)=f(H(z))$, and

c. for all $f$ in $A, T f=\sup \{|(T f)(x)| ; x$ in $S\}$.

Proof. Let $B=T(A)=\{f ; f=T g$ for some $g$ in $A\}$.

$B$ is a closed subspace of the Banach space of continuous complex valued functions on the unit circle and $T$ is an isometry of $A$ onto $B$. Hence $T^{*}$, the adjoint of $T$, is an isometry of $B^{*}$ onto $A^{*}$. Thus each extreme point of the unit ball of $A^{*}$ is the image under $T^{*}$ of an extreme point of the unit ball of $B^{*}$.

For $x$ a point of $C$ and $\alpha$ a complex number we denote by $\alpha e_{x}$ the element of $A^{*}$ such that $\left(\alpha e_{x}\right)(f)=\alpha f(x)$. The extreme points of the unit ball of $A^{*}$ are exactly all those functionals of the form $\alpha e_{x}$ for $\alpha$ of modulus one. Furthermore, the extreme points of the unit ball of $B^{*}$ are all of the form $\alpha e_{x}$ for $\alpha$ of modulus one; although not all such functionals are extreme points. Let $R$ be the set of points, $x$, in $C$ such that $e_{x}$ is an extreme point of the unit ball of $B^{*}$. Let $S$ be the closure of $R$ and let $H$ be the function $T(Z)$ restricted to the set $S$. We know that for any $x$ in $R, T^{*}\left(e_{x}\right)=$ $\alpha(x) e_{t(x)}$ where $\alpha(x)$ is a scalar of modules one and $t(x)$ is a point of $C$. Hence, for an $f$ in $A$ and any $x$ in $R$

$$
(T f)(x)=\alpha(x) f(t(x)) \text {. }
$$

By evaluating (1) with $f$ equal to the constant function, 1, we see 
that $\alpha(x)=1$. Evaluating (1) with $f=Z$ we see that $t(x)=H(x)$. Hence for all $f$ in $A$ and all $x$ in $R$, $(T f)(x)=f(H(x))$. Since all of the functions in $A$ are continuous on $C$ and $H$ can be extended to be continuous on $S$, this last equation holds at all points of $S$.

It remains to verify the third part of the conclusion of the theorem. For any $f$ in $A,\|T f\|=\|f\|$ and $\|f\|=|f(y)|$ for some $y$ in $C . \quad e_{y}$ is an extreme point of the unit ball of $A^{*}$, hence there is a point $x$ in $R$ such that $T^{*}\left(e_{x}\right)=e_{y}$. Hence $y=H(x)$ and $\|T f\|=$ $|f(y)|=|f(H(x))|=|T f(x)| \leqq \sup \{|T f(w)| ; w$ in $S\}$. The reverse inequality is immediate and the theorem is proved.

We note in passing that from the proof it is clear that this theorem is merely an instance of a general function algebra result.

COROLLARY. In the situation of the previous theorem, if the Lebesgue measure of the set $S$ is positive then $T$ is in $K^{\prime}$.

Proof. By the theorem, for any $f$ in $A$, the functions $T f$ and $f \circ T(Z)$ agree on the set $S$. If two functions in $A$ agree on a subset of $C$ of positive measure they are equal. Hence $T f=f \circ T(Z)$ and thus $T$ is in $K^{\prime}$.

Corollary. Theorem $A$ is true.

Proof. Pick $f$ and $g$ in $A$. Consider the function $h=T(f g)-$ $T(f) T(g)$. Since $T(A)$ is a ring, $h$ is in $T(A)$ and thus $h=T(k)$ for some $k$ in $A$. By the previous theorem $\|h\|=\|T k\|=\sup \{|T k(x)|$; $x$ in $S$, where $S$ is the set obtained in the previous theorem. But the previous theorem also shows that for $k$ in $S$,

$T k(x)=T(f g)(x)-T f(x) T g(x)=(f g)(T(Z)(x))-f(T(Z)(x)) g(T(Z)(x))=0$.

Hence $\|h\|=0$. So $h=0$ and thus $T(f g)=T(f) T(g)$.

An example in the last section shows that the hypothesis that $T(A)$ be a ring cannot be omitted.

3. Linear maps which take functions of norm one to extreme points. In this section we will show that if $T f(z)$ is large (compared to $\|f\|)$ then for any $g$ in $A,(T f g-T f T g)(z)$ is small. The estimates developed will allow us to conclude, in some cases, that $T(f g)-$ $T(f) T(g)$ is identically zero. Given $T$ in $K$ we define $U$ mapping $A \times$ $A$ to $A$ by $U(f, g)=T(f g)-T(f) T(g)$.

Theorem. Given $T$ in $K, f$ and $g$ in $A$, $\|f\| \leqq 1$ and $\|g\| \leqq 1$, and $z$ in $C$ then $|U(f, g)(z)| \leqq 4(1-|T f(z)|)^{1 / 4}$. 
Proof. Without loss of generality we can assume that $\operatorname{Tf}(z)=$ $d$, a nonnegative real number. The linear functional, $L$, on $A$ defined by $L(h)=T h(z)$ for all $h$ in $A$ is of norm one and $L(1)=1$. Hence there is a probability measure, $\mu$, on $C$, the boundary of $D$, such that for all $h$ in $A, T h(z)=L(h)=\int h d \mu$. Set $S=\{x ; x$ in $C$ and $\operatorname{Re}(f(x)) \leqq$ $d-\sqrt{1-d}\}$. Since $d$ and $\mu$ are real we have $d=\int \operatorname{Re}(f) d \mu$. Hence $d=\int_{S} \operatorname{Re}(f) d \mu+\int_{C-S} \operatorname{Re}(f) d \mu$. So $d \leqq \mu(S)(d-\sqrt{1-d})+\mu(C-S)=$ $\mu(S)(d-1-\sqrt{1-d)}+1$. Hence $\mu(S) \leqq(1-d) /(1-d+\sqrt{1-d}) \leqq \sqrt{1-d}$. Thus

$$
\begin{aligned}
|U(f, g)(z)| & =|T(f g)(z)-T f(z) T g(z)| \\
& =\left|\int f g d \mu-\left(\int f d \mu\right)\left(\int g d \mu\right)\right| \\
& =\left|\int f g d \mu-d \int g d \mu\right| \\
& =\left|\int(f-d) g d \mu\right| \\
& \leqq\left|\int_{S}(f-d) g d \mu\right|+\left|\int_{C-S}(f-d) g d \mu\right| \\
& \leqq \mu(S)\|f-d\|\|g\|+\|g\| \sup \{|f(x)-d| ; x \text { in } C-S\} .
\end{aligned}
$$

But on $C-S,|f(x)-d|^{2} \leqq(\sqrt{1-d})^{2}+\left(1-(d-\sqrt{1-d})^{2}\right)$. Hence, on $C-S,|f(x)-d| \leqq 4(1-d)^{1 / 4}$. Thus

$$
\begin{aligned}
|U(f, g)(z)| & \leqq 4(1-d)^{1,2}+2(1-d)^{1 / 4} \\
& <6(1-d)^{1 / 4}
\end{aligned}
$$

and the theorem is proved.

In order to use this result we recall two facts about the algebra $A$. Let $d \theta$ be normalized Lebesgue measure on $C$. An element $f$ of $A$ is the zero function if and only if $\int_{C} \log \left|f\left(e^{i \theta}\right)\right| d \theta=-\infty$ and $f$ in $A$ is an element of $L$ if and only if $\int_{C} \log \left(1-\left|f\left(e^{i \theta}\right)\right|\right) d \theta=-\infty$.

Corollary. If $T$ is in $K$ and $f$ in $A$ with $\|f\|=1$ and if $T f$ is in $L$, then for all $g$ in $A, T(f g)=T(f) T(g)$.

Proof. It suffices to show that for any $g$ of norm one, $U(f, g)$ is the zero function. By the previous theorem

$$
\int_{C} \log \left|U(f, g)\left(e^{i \theta}\right)\right| d \theta \leqq \int_{C} \log (6)+\frac{1}{4} \log \left(1-\left|T f\left(e^{i \theta}\right)\right|\right) d \theta .
$$


By hypothesis $T f$ is extreme, hence the integral on the right hand side of the inequality is $-\infty$. Thus, by the previous comments $U(f, g)$ is the zero function. The proof is complete.

For any elements $h$ in $A$, we denote by $[1, h]$ the smallest closed subalgebra of $A$ which contains the elements 1 and $h$. Thus $[1, h]$ is the closure in $A$ of the space of polynomials in $h$.

Corollary. If $T$ is in $K$ and $f$ in $A$ with $\|f\|=1$ and if $T f$ is in $L$, then $T$ is multiplicative when restricted to the algebra generated by $f$ and the constants. That is, for all $g$ and $g^{\prime}$ in $[1, f]$, $T\left(g g^{\prime}\right)=T(g) T\left(g^{\prime}\right)$.

Proof. By the continuity of $T$, it suffices to show that $T\left(f^{n}\right)=$ $T(f)^{n}$ for all positive integers, $n$. If $n=1$ there is nothing to prove. Hence a proof by induction is immediate using the previous corollary for the induction step.

Corollary. If $T$ is in $K$ and $f$ in $A$ with $\|f\|=1$ and if $T f$ is in $L$ and if $f$ is univalent on $D$, then $T$ is in $K^{\prime}$.

Proof. By the previous corollary it suffices to show that if $f$ is univalent on $D$ then $[1, f]=A$. Let $F$ be the function on $f(D)$ which is the inverse function of $f, F \circ f=Z$. Mergelyan's theorem applies to $f(D)$, hence there are polynomials, $P_{n}$, which, on $f(D)$, converge uniformly to $F$. Hence the functions $P_{n} \circ f$ are elements of $[1, f]$ which, on $D$, converge uniformly to $Z$. Thus $Z$ is in $[1, f]$ and hence $[1, f]=A$.

\section{COROLlaRy. Theorem $B$.}

4. Notes and a conjecture. In this section we offer a number of examples to indicate that the above results are, in various senses, the best possible. In all cases the verification that the examples have the properties attributed to them is straightforward and is omitted.

Let $S$ be a closed subset of $C$ of Lebesgue measure zero and homeomorphic to a Cantor set. Let $H$ be a continuous mapping of $S$ onto $C$. Rudin's theorem guarantees that we can find a function $h_{1}$ in $A$ and of norm one such that for any $x$ in $S, h_{1}(x)=H(x)$. By adjoining a point to $S$ and applying Rudin's theorem again we can find $h_{2}$ in $A$ and of norm one so that for all $x$ in $S, h_{2}(x)=H(x)$ and $h_{1} \neq h_{2}$. We will consider the following elements of $K$ : 


$$
T_{1} f(z)=\frac{1}{2}\left(f\left(h_{1}(z)\right)+f\left(h_{2}(z)\right), T_{2} f(z)=\frac{1}{2}(f(z)+f(-z)),\right.
$$

and $T_{3} f(z)=(f(z)+f(a z)) / 2$ with a nonzero constant of modulus less than one.

$T_{1}$ is in $K . T_{1}$ is an isometry, and $T_{1}$ is not in $K^{\prime}$. Hence the hypothesis in Theorem $\mathrm{A}$ that $T(A)$ be a ring cannot be dropped. Not all isometries in $K$ are multiplicative. ${ }^{1}$

$T_{2}$ is in $K$ and $T_{2}\left(Z^{2}\right)=Z^{2}$, but $T_{2}$ is not in $K^{\prime}$. Thus Theorem $\mathrm{B}$ is no longer true if the restriction that $T(Z)$ be in $L$ is replaced by the restriction that $T\left(Z^{n}\right)$ be in $L$ for some or even for infinitely many $n$.

$T_{3}$ is in $K$ and $T_{3}$ is surjective and invertible but $T_{3}$ is not in $K^{\prime}$. Thus the hypothesis in Theorem A that $T$ be an isometry cannot be weakened to the hypothesis that the ratio $\|f\| /\|T f\|$ be bounded.

It is not clear that the exponent $1 / 4$ in the theorem of the previous section is the best possible. If $T$ in $K$ is an average of two elements of $K^{\prime}$ (for example $T=T_{1}$ ) then a direct estimate shows that the exponent can be replaced by $1 / 2$. On the other hand, it is not clear that even such a stronger result with exponent of $1 / 2$ would lead to a simple proof of the following.

Conjecture. The extreme points of $K$ all lie in $K^{\prime} .^{2}$

It follows from the corollary in the introduction that this conjecture is equivalent to the conjecture that if $T$ is an extreme point of $K$ then $T(Z)$ is in $L$.

\section{REFERENCES}

1. Frank T. Birtel, ed., Function Algebras, Proceedings of an International Symposium on Function Algebras held at Tulane University, 1965, Scott, Foresman and Co., Glenview, Ill., 1966.

2. Kenneth Hoffman, Banach Spaces of Analytic Functions, Prentice-Hall Inc., Englemood Cleffs, N. J., 1962.

3. R. Phelps, Extreme positive operators and homeomorphisms, Trans. Amer, Math. Soc., 108 (1963), 265-274.

Received February 4, 1971. Some of the results in this paper were contained in the author's doctoral dissertation written under the guidance of Professor A. Gleason (Harvard, 1970).

\section{WASHINGTON UNIVERSITY}

1 This example is known to a number of people and was communicated to the author by J. Ryff and by F. Forelli.

2 It has since been pointed out to the author that this conjecture is false. ("Extreme Nonmultiplicative Operators", by J. Lindenstrauss, R. R. Phelps, and J. V. Ryff, unpublised.) 


\section{PACIFIC JOURNAL OF MATHEMATICS}

\section{EDITORS}

H. SAMELSON

Stanford University

Stanford, California 94305

C. R. Hовву

University of Washington

Seattle, Washington 98105
J. DUGUNDJI

Department of Mathematics

University of Southern California

Los Angeles, California 90007

RICHARD ARENS

University of California

Los Angeles, California 90024

\section{ASSOCIATE EDITORS}
E. F. BECKENBACH
B. H. NeumanN
F. WOLF
K. YoSHIDA

\section{SUPPORTING INSTITUTIONS}

UNIVERSITY OF BRITISH COLUMBIA

CALIFORNIA INSTITUTE OF TECHNOLOGY

UNIVERSITY OF CALIFORNIA

MONTANA STATE UNIVERSITY

UNIVERSITY OF NEVADA

NEW MEXICO STATE UNIVERSITY

OREGON STATE UNIVERSITY

UNIVERSITY OF OREGON

OSAKA UNIVERSITY

UNIVERSITY OF SOUTHERN CALIFORNIA
STANFORD UNIVERSITY

UNIVERSITY OF TOKYO

UNIVERSITY OF UTAH

WASHINGTON STATE UNIVERSITY

UNIVERSITY OF WASHINGTON

AMERICAN MATHEMATICAL SOCIETY CHEVRON RESEARCH CORPORATION NAVAL WEAPONS CENTER 


\section{Pacific Journal of Mathematics}

\section{Vol. 38, No. $1 \quad$ March, 1971}

Bruce Alan Barnes, Banach algebras which are ideals in a Banach algebra ..... 1

David W. Boyd, Inequalities for positive integral operators............... 9

Lawrence Gerald Brown, Note on the open mapping theorem .............. 25

Stephen Daniel Comer, Representations by algebras of sections over Boolean

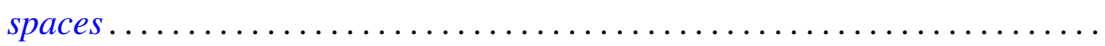

John R. Edwards and Stanley G. Wayment, On the nonequivalence of

conservative Hausdorff methods and Hausdorff moment sequences ........

P. D. T. A. Elliott, On the limiting distribution of additive functions $(\bmod 1) \ldots \ldots$

Mary Rodriguez Embry, Classifying special operators by means of subsets

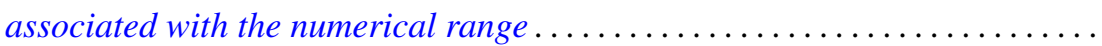

Darald Joe Hartfiel, Counterexamples to a conjecture of G. N. de Oliveira ......

C. Ward Henson, A family of countable homogeneous graphs...............

Satoru Igari and Shigehiko Kuratsubo, A sufficient condition for

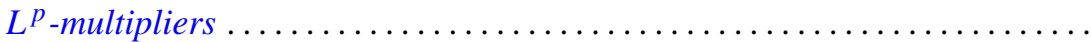

William A. Kirk, Fixed point theorems for nonlinear nonexpansive and

generalized contraction mappings............................

Erwin Kleinfeld, A generalization of commutative and associative rings ...... 95

D. B. Lahiri, Some restricted partition functions. Congruences modulo $11 \ldots \ldots 103$

T. Y. Lin, Homological algebra of stable homotopy ring $\pi *$ of spheres ....... 117

Morris Marden, A representation for the logarithmic derivative of a meromorphic function...........................

John Charles Nichols and James C. Smith, Examples concerning sum properties for metric-dependent dimension functions . .

Asit Baran Raha, On completely Hausdorff-completion of a completely

Hausdorff space.

M. Rajagopalan and Bertram Manuel Schreiber, Ergodic automorphisms and affine transformations of locally compact groups..........

N. V. Rao and Ashoke Kumar Roy, Linear isometries of some function

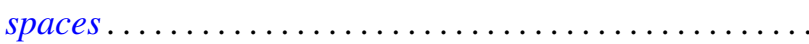

William Francis Reynolds, Blocks and F-class algebras of finite groups

Richard Rochberg, Which linear maps of the disk algebra are multiplicative ...

Gary Sampson, Sharp estimates of convolution transforms in terms of decreasing

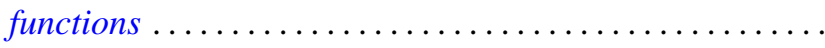

Stephen Scheinberg, Fatou's lemma in normed linear spaces

Ken Shaw, Whittaker constants for entire functions of several complex

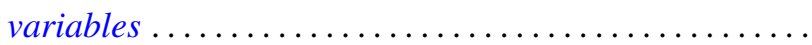

James DeWitt Stein, Two uniform boundedness theorems................ 251

$\mathrm{Li} \mathrm{Pi} \mathrm{Su,} \mathrm{Homomorphisms} \mathrm{of} \mathrm{near-rings} \mathrm{of} \mathrm{continuous} \mathrm{functions} \mathrm{.} \mathrm{.............} 261$

Stephen Willard, Functionally compact spaces, $C$-compact spaces and mappings of minimal Hausdorff spaces....................... 